\title{
Rehabilitación respiratoria en pacientes EPOC: experiencia en Atención Primaria de Salud
}

\author{
ALEX CAMPOS A.*, OSVALDO CABRERA R.** y FRANCISCO ARANCIBIA H.***
}

\section{Respiratory rehabilitation in COPD patients: Experience in a rural Primary Health Care Center}

Respiratory rehabilitation improves physical capacity and quality of life in COPD patients as it has been previously reported. In Chile there are few and unpublished experiences on respiratory rehabilitation programs (RRP) implemented in primary health care (PHC). Our aim was to evaluate RRP outcome in COPD patients carried out in a rural PHC center. Methods: A prospective descriptive study of RRP in a PHC center implemented in Talagante (a village located $40 \mathrm{~km}$ from Santiago). COPD patients were enrolled in the study from July 2012 to May 2014. Patients having the inclusion criteria were admitted to a 12 weeks-long RPP. This program included a 90 minute-long sessions of aerobic training (walking), strength training of upper and lower limbs (dumbbell, ankle support and elastic bands) and respiratory muscle training (threshold valve), twice a week. Quality of life, strength of trained muscles, six-minutes walking test (TM6), BODE index and dyspnea index, were measured before and after completing RRP. Results were expressed as mean $\pm S D$. Results: Out of 79 patients initially assessed, 54 were admitted to the RRP and 39 patients completed the program and were included in the analysis. Their mean age was $67.3 \pm 8.5$ years, $64 \%$ were female. In average BMI was $26.97 \pm 4.3$ $\mathrm{kg} / \mathrm{m}^{2}$, and $\mathrm{FEV} V_{1}$ was $1.17 \pm 0.57$ liters. We demonstrated a statistically significant improvement after RRP in 6-minute walk test (445.9 $\pm 77.9 \mathrm{~m}$ versus $498.2 \pm 82.8 \mathrm{~m}, p<0.001)$; Saint George's Respiratory Questionnaire score (45.1 \pm 16.1 versus $18.5 \pm 11.5, p<0.0001)$, PIMax (48.95 \pm 18.8 versus $\left.57.2 \pm 19.4 \mathrm{~cm} \mathrm{H}_{2} \mathrm{O}, p<0.0001\right), \mathrm{BODE}$ index (2.76 \pm 1.37 versus $\left.1.53 \pm 1.0, p<0.0001\right)$, and $\mathrm{mMRC}$ dyspnea scale $(2.54 \pm 0.75$ versus $1.18 \pm 0.56, p<0.0001)$. Conclusions: Our results are consistent with those reported in the literature, demonstrating that COPD patients get beneficial effects with the respiratory rehabilitation program. We also showed that RRP can be implemented in primary health care using simple tools, with reasonable costs.

Key words: COPD; Respiratory Rehabilitation; Primary health care.

\section{Resumen}

La rehabilitación respiratoria ha demostrado beneficios en mejorar la capacidad fisica y calidad de vida en los pacientes con EPOC. Sin embargo, en Chile no existe información de programas de rehabilitación respiratoria (PRR) en la atención primaria de salud. El objetivo de nuestro trabajo fue evaluar los resultados de la implementación de un programa de rehabilitación respiratoria (PRR) en pacientes con EPOC en la atención primaria de salud. Material y Método: Estudio prospectivo descriptivo realizado en un centro de salud familiar de Talagante en pacientes con diagnóstico confirmado de EPOC, durante el periodo julio de 2012 a mayo de 2014. Fueron ingresados al PRR, de 12 semanas de duración, aquellos pacientes que cumplían los criterios de inclusión. Se realizó entrenamiento aeróbico (caminata), entrenamiento de fuerza de extremidades superiores e inferiores (mancuernas, tobilleras y bandas elásticas) y entrenamiento de musculatura respiratoria (válvula umbral), 2 veces por semana y de 90 min de duración cada sesión. Al iniciar y al finalizar el PRR se evaluó: calidad de vida, fuerza

\footnotetext{
* Kinesiólogo, Centro de Salud Familiar Dr. Alberto Allende Jones, Talagante.

** Kinesiólogo, Instituto Nacional del Tórax.

*** Médico, Servicio de Medicina Respiratoria, Instituto Nacional del Tórax.
} 
muscular, test de marcha de 6 min (TM6); indice BODE y disnea. Resultados: De un total de 79 pacientes con EPOC evaluados, 54 ingresaron al PRR y 39 finalizaron el programa. La edad promedio fue $67,3 \pm 8,5$ años, $64 \%$ de género femenino, indice de mas a corporal: $26,97 \pm 4,3 \mathrm{~kg} / \mathrm{m}^{2}$ y $\mathrm{VEF} \mathrm{F}_{1} 1,17$ $\pm 0,57$ L. Al comparar los valores iniciales y finales del PRR se encontraron diferencias estadísticamente significativas en: TM6 (445,9 $\pm 77,9$ vs 498,2 $\pm 82,8 \mathrm{~m}, p<0,001)$; Puntaje del cuestionario de St. George (45,1 $\pm 16,1$ vs 18,5 $\pm 11,5, p<0,0001)$, PIMáx 48,95 $\pm 18,8$ vs 57,2 $\pm 19,4 \mathrm{~cm} \mathrm{H}_{2} \mathrm{O}, p<$ $0,0001)$, indice BODE (2,76 $\pm 1,37$ vs $1,53 \pm 1,0, p<0,0001)$, y escala de disnea mMRC $(2,54 \pm 0,75$ vs 1,18 $\pm 0,56, p<0,0001)$. Conclusiones: Los resultados de nuestro estudio son concordantes con lo descrito en la literatura y demuestran los beneficios significativos obtenidos con la rehabilitación respiratoria en pacientes con EPOC. También, se demuestra que en Chile se puede implementar un PRR en la atención primaria utilizando implementos simples y con un bajo costo.

Palabras clave: EPOC; Rehabilitación Respiratoria; Atención Primaria.

\section{Introducción}

En Chile y el mundo, la enfermedad pulmonar obstructiva crónica (EPOC) es un problema de salud pública, debido a su alta prevalencia, su condición progresiva, el deterioro de la calidad de vida y el gran impacto económico ${ }^{1}$. Así también, se proyecta que la EPOC será la tercera causa más frecuente de muerte en el mundo para el año $2020^{2}$. Según la Organización Mundial de la Salud se estima que más de 80 millones de personas en el mundo padecen de EPOC moderada a severa $^{3}$. La prevalencia mundial de EPOC en todas las edades en el año de 2002 fue estimada en 11,6 por 1.000 en hombres y 8,8 por 1.000 en mujeres. En América Latina, la prevalencia de la EPOC en la población adulta oscila entre el 8 y $20 \%{ }^{4}$. En Chile, el estudio PLATINO reportó una prevalencia de $16,9 \%{ }^{5}$. La rehabilitación respiratoria (RR) es un tratamiento multidisciplinario y en los pacientes con EPOC permite intervenir el círculo vicioso que limita la capacidad de ejercicio ${ }^{1,4}$. En la actualidad se puede afirmar con un alto nivel de evidencia científica que los PRR mejoran la disnea, la capacidad de ejercicio y la calidad de vida en pacientes EPOC $^{6-8}$, constituyéndose en la piedra angular en el manejo de estos pacientes. Existe controversia en relación a la etapa de la EPOC en que se debe iniciar la rehabilitación. Berry y cols. ${ }^{9}$, encontraron que todos los pacientes con EPOC (leve, moderada y severa) se beneficiaron con un programa de rehabilitación respiratoria. El estudio de Takigawa y cols. ${ }^{10}$, también encontró que los pacientes con EPOC se beneficiaron de un PRR independientemente de la etapa de la enfermedad.

En Chile, existe escasa información sobre PRR en pacientes EPOC en la atención primaria. El objetivo de nuestro trabajo fue evaluar un programa de rehabilitación respiratoria realizado en la atención primaria, en una zona rural.

\section{Material y Método}

Estudio prospectivo descriptivo realizado en el Centro de Salud Familiar (CESFAM), "Dr. Alberto Allende Jones" de Talagante, Región Metropolitana, durante el período comprendido entre julio de 2012 y mayo de 2014. Fueron incluidos en forma consecutiva los pacientes con diagnóstico confirmado de EPOC según criterios AUGE (acceso universal de garantías explícitas) ${ }^{11}$, y que fueron derivados a la sala ERA (Enfermos Respiratorios Adultos). Los criterios de exclusión incluyeron: comorbilidades descompensadas, patología osteomuscular que impidiese la actividad física, inadecuado nivel cognitivo, falta de motivación o falta de disponibilidad para participar en el programa.

A todos los pacientes se les explicó el objetivo del programa. La rehabilitación respiratoria está incluida dentro de las "Garantías Explícitas en Salud" (GES), y forma parte integral de los cuidados del paciente con EPOC ${ }^{11}$. Al ingreso al PRR se requirió un consentimiento informado escrito para obtener la autorización del paciente para la recolección de datos. El estudio fue aprobado por la Dirección del CESFAM Dr. Alberto Allende Jones de Talagante.

El Programa de Rehabilitación Respiratoria tuvo una duración de 12 semanas y se realizó en grupos de 8 a 10 pacientes. El equipo de salud estaba constituido por un kinesiólogo y un médico de atención primaria.

Se evaluó al iniciar y al finalizar el PRR las siguientes variables: 1) Calidad de vida relacionada con la salud, utilizando el cuestionario de Saint George (CSG) ${ }^{12}$; 2) Fuerza muscular respiratoria, utilizando pimómetro (manómetro para medir PIMáx) y flujómetro ${ }^{13}$; 3 ) Capacidad física, utilizando el test de marcha de 6 min (TM6) ${ }^{14}$; 4) Predicción de mortalidad utilizando el Índice BODE $^{15}$ y 5) Disnea, utilizando la escala del Me- 
dical Research Council modificada (mMRC) ${ }^{16}$. Además, los pacientes recibieron una primera sesión consistente en una charla educativa y motivacional, donde se les enseñó el uso correcto de inhaladores, conocimiento de la enfermedad, beneficios de los programas de rehabilitación y experiencias previas de otros pacientes. Posteriormente, se realizó entrenamiento aeróbico, entrenamiento de fuerza de extremidades superiores (EESS) e inferiores (EEII), y entrenamiento de musculatura respiratoria, dos veces por semana y de 90 min de duración cada sesión (Tabla 1). Cada paciente realizó dos sesiones semanales, durante 3 meses, completando un total de 24 sesiones. En los pacientes usuarios de oxígeno domiciliario, se mantuvo el flujo de $\mathrm{O}_{2}$ durante el entrenamiento. No se utilizó oxígeno adicional en los pacientes que no eran oxígeno-dependientes. Los materiales utilizados fueron: pimómetros y válvulas umbral de entrenamiento, flujómetro de Wright, bandas elásticas, tobilleras, mancuernas, globos, vasos y bombillas, pasillo y escaleras del establecimiento.

Se registraron datos demográficos, espirométricos, hábito tabáquico, comorbilidades, índice de masa corporal (IMC), severidad de la enfermedad para la cual se utilizó la clasificación de GOLD $2011^{17}$. Además, las siguientes variables se registraron al iniciar y finalizar el programa de entrenamiento: distancia recorrida, PIMáx, flujometría, puntaje del CSG, Índice de Masa Corporal (IMC), escala de disnea mMRC, e índice BODE.

\section{Análisis estadístico}

Los resultados son expresados como promedios \pm DS. Se utilizó para variables continuas la prueba de t-Student, y para variables categóricas la prueba de Chi-cuadrado o prueba de Fisher. Los datos iniciales y post entrenamiento, de los pacientes que completaron el PRR, fueron comparados usando test t-Student para muestras pareadas. El nivel de significación se estableció en 0,05. Para el análisis de los datos se utilizó el programa estadístico IMB SPSS versión 22.

\section{Resultados}

De un total de 294 pacientes con EPOC en control en el CESFAM de Talagante, 79 fueron evaluados durante este período y 25 fueron excluidos por las siguientes causas: no aceptaron participar (9 pacientes), dificultad logística (7 pacientes), patología cardiovascular descompensada (4 pacientes), alteración cognitiva (3 pacientes) y patología osteomuscular severa (2 pacientes). Ingresaron al PRR, un total de 54 pacientes que cumplían los criterios de inclusión; sus características se describen en la Tabla 2. Del total de pacientes incluidos, 39 (72\%) finalizaron el programa. Las causas por las cuales el $28 \%$ de los pacientes no completó el PRR se describen en la Figura 1. La edad promedio fue $67,3 \pm 8,5$ años, $64 \%$ de género femenino, índice de masa corporal de $27 \pm 4,3$ y $\mathrm{VEF}_{1}=1,17 \pm 0,57 \mathrm{~L}(59,8$ $\pm 21 \%$ ). Cincuenta y seis porciento de los casos

Tabla 1. Características de las sesiones de entrenamiento

1. Evaluación signos vitales

- Presión arterial; frecuencia respiratoria; frecuencia cardíaca; oximetría de pulso

2. Evaluación de la terapia inhalatoria

$5 \min$

5 min

3. Fase calentamiento

$20 \mathrm{~min}$

4. Entrenamiento aeróbico

- Caminata al 70-80\% de la carga inicial del TM6, 6 min de intensidad programada intercalado con pausas de $2 \min , 6-2-6$

5. Entrenamiento de la fuerza

- EEII: Bandas elásticas-tobilleras (2-3 Series) con 8-10 repeticiones

$10 \mathrm{~min}$

- EESS: Bandas elásticas-mancuernas (2-3 Series) con 8-10 repeticiones

$10 \mathrm{~min}$

6. Entrenamiento musculatura respiratoria:

- Válvula de entrenamiento: 30\% PIMáx

$15 \mathrm{~min}$

- Actividades lúdicas: inflar globos, vaso con agua y bombillas, etc

$10 \mathrm{~min}$

7. Fase relajación (música) $5 \mathrm{~min}$

8. Evaluación final 
Tabla 2. Características generales de los pacientes con EPOC que fueron seleccionados y los que completaron el Programa de Rehabilitación Respiratoria (PRR)

\begin{tabular}{|c|c|c|}
\hline Características & $\begin{array}{l}\text { Pacientes seleccionados } \\
\qquad(\mathrm{n}=\mathbf{5 4})\end{array}$ & $\begin{array}{l}\text { Pacientes completaron PRR } \\
\qquad(\mathrm{n}=\mathbf{3 9})\end{array}$ \\
\hline Edad, años* & $66,6 \pm 9,4$ & $67,3 \pm 8,5$ \\
\hline Género Femenino, \% & 61,4 & 64 \\
\hline $\mathrm{VEF}_{1}, \mathrm{ml}(\%)^{*}$ & $1.235 \pm 607(60,5 \pm 22)$ & $1.170 \pm 570(59,8 \pm 21)$ \\
\hline $\mathrm{CVF}, \mathrm{ml}(\%)^{*}$ & $2.280 \pm 722(79,4 \pm 21)$ & $2.261 \pm 690(78 \pm 20,3)$ \\
\hline Antecedente Tabáquico, n (\%) & $54(100)$ & $39(100)$ \\
\hline \multicolumn{3}{|l|}{ Comorbilidades, $\mathrm{n}(\%)$} \\
\hline - Hipertensión arterial & $41(76)$ & $31(79)$ \\
\hline - Diabetes mellitus & $16(30)$ & $9(23)$ \\
\hline - Artrosis leve de rodilla & $11(20)$ & $8(21)$ \\
\hline - Dislipidemia & $7(13)$ & $5(13)$ \\
\hline - Insuficiencia cardíaca & $6(11)$ & $3(8)$ \\
\hline - Hipotiroidismo & $3(6)$ & $2(5)$ \\
\hline $\mathrm{IMC}, \mathrm{kg} / \mathrm{m}^{2 *}$ & $27,4 \pm 5,1$ & $26,9 \pm 4,3$ \\
\hline \multicolumn{3}{|l|}{ Clasificación GOLD**, n (\%) } \\
\hline - $\operatorname{GOLD~I}\left(\mathrm{VEF}_{1} \geq 80 \%\right)$ & $11(20,4)$ & $8(20,5)$ \\
\hline - GOLD II $\left(\mathrm{VEF}_{1}<80 \%\right)$ & $20(37)$ & $14(35,9)$ \\
\hline - GOLD III $\left(\mathrm{VEF}_{1}<50 \%\right)$ & $19(35,2)$ & $15(38,4)$ \\
\hline - GOLD IV $\left(\mathrm{VEF}_{1}<30 \%\right)$ & $4(7,4)$ & $2(5,2)$ \\
\hline Oxígenoterapia domiciliaria, n (\%) & $3(5,3)$ & $2(5,2)$ \\
\hline
\end{tabular}

*Promedio \pm desviación estándar. **Lacasse et al ${ }^{8}$.

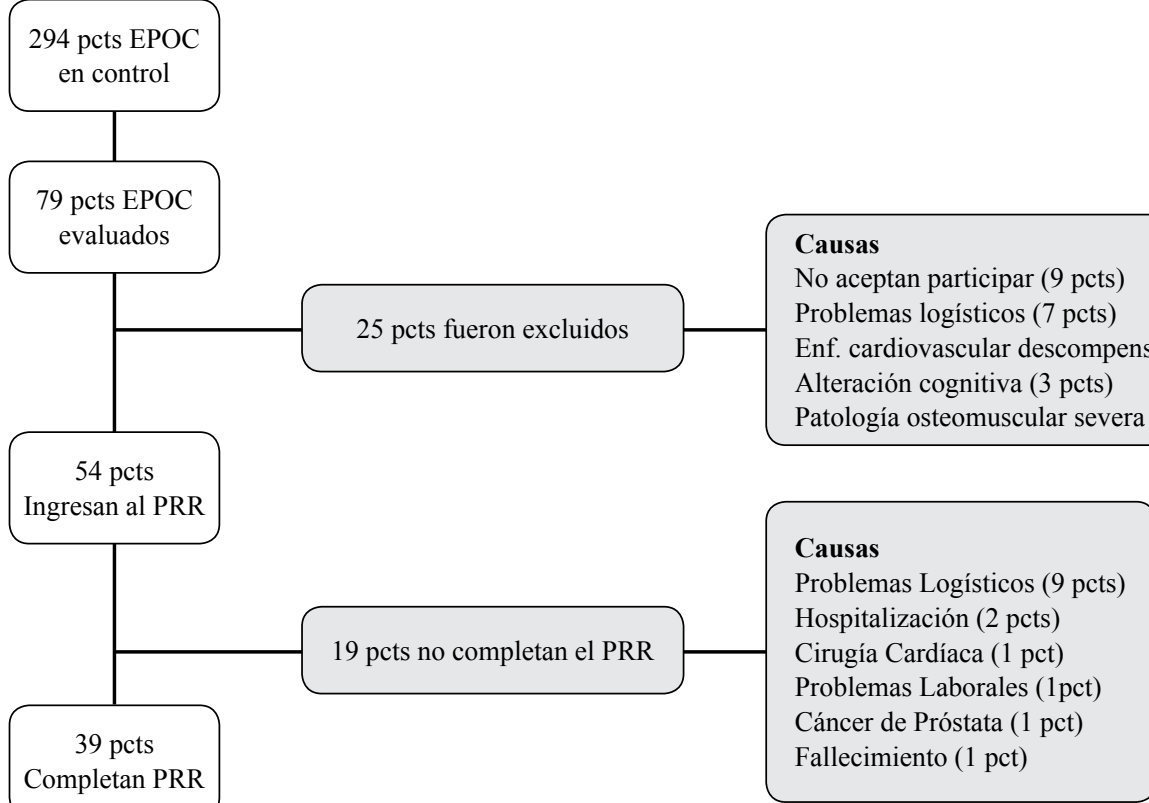

Figura 1. Algoritmo de selección, ingreso y cumplimiento del Programa de Rehabilitación Respiratoria (PRR) en la atención primaria de pacientes (pcts) con EPOC. 
Tabla 3. Análisis comparativo de las variables capacidad física, disnea, índice de masa corporal, índice BODE, fuerza muscular respiratoria y calidad de vida, antes y al finalizar el programa de rehabilitación respiratoria en 39 pacientes con EPOC

\begin{tabular}{|lccc|}
\hline Variable & Basal & Post-Entrenamiento & p \\
TM6, m & $445,9 \pm 77,9$ & $498,2 \pm 82,8$ & $<0,0001$ \\
mMRC, puntos & $2,5 \pm 0,7$ & $1,2 \pm 0,5$ & $<0,0001$ \\
IMC, $\mathrm{kg} / \mathrm{m}^{2}$ & $26,9 \pm 4,4$ & $26,8 \pm 4,3$ & 0,11 \\
BODE, puntos & $2,8 \pm 1,4$ & $1,5 \pm 1,0$ & $<0,0001$ \\
PIMáx, cmH${ }_{2} \mathrm{O}$ & $48,9 \pm 18,8$ & $57,2 \pm 19,4$ & $<0,0001$ \\
Flujometría, L/min & $268,2 \pm 110,7$ & $286,1 \pm 113,2$ & 0,06 \\
CSG, puntos & $45,1 \pm 16,0$ & $18,5 \pm 11,5$ & $<0,0001$ \\
\hline
\end{tabular}

Las cifras de la tabla representan el valor promedio de cada variable \pm su desviación estándar. TM6: Test de marcha de 6 min; mMRC: Escala de disnea del Medical Research Council modificada; IMC: índice de masa corporal; BODE: B-body mass, O-obstrucción, D-disnea, E-capacidad de ejercicio ${ }^{15}$. PIMáx: presión inspiratoria máxima. CSG: calidad de vida según cuestionario de St. George.

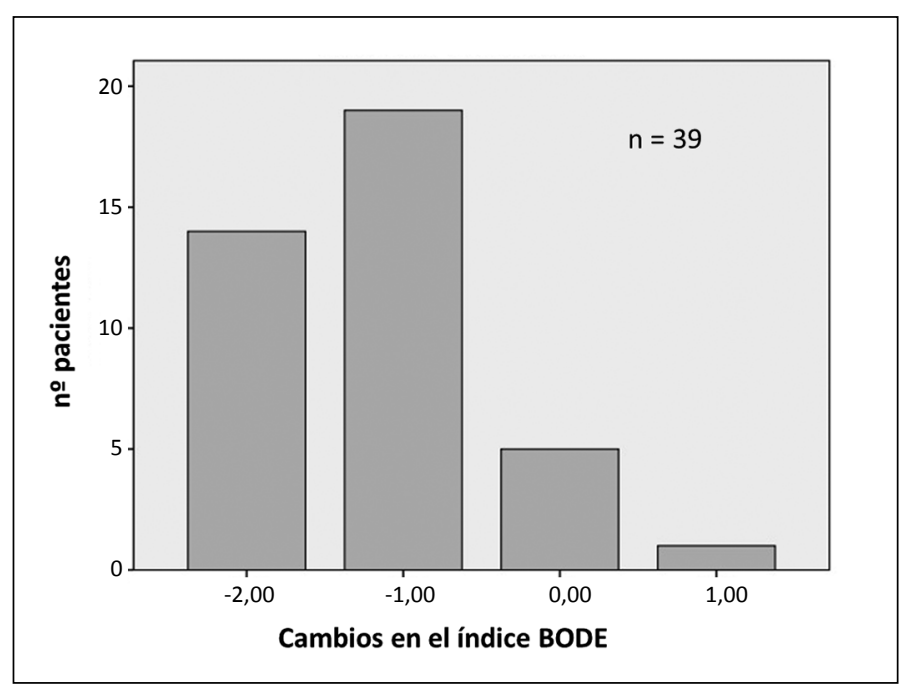

Figura 2. Distribución del cambio en el índice BODE en los 39 pacientes con EPOC que completaron el programa de rehabilitación respiratoria. La mejoría del índice se manifiesta mediante disminuciones de la puntuación, mientras que el aumento de su valor implica un peor pronóstico. correspondían a categoría GOLD leve o moderado (I o II) y las principales comorbilidades fueron la hipertensión arterial y la diabetes mellitus.

Al comparar los valores de distintas variables al iniciar y finalizar el PRR (Tabla 3), se encontraron diferencias estadísticamente significativas en las siguientes mediciones: distancia recorrida del TM6 $(445,9 \pm 77,9$ vs 498,2 $\pm 82,8$ $\mathrm{m} ; \mathrm{p}<0,001)$; puntaje CSG $(45,1 \pm 16,1$ vs $18,5 \pm 11,5 ; \mathrm{p}<0,0001)$, PIMáx 48,95 $\pm 18,8$ vs $\left.57,2 \pm 19,4 \mathrm{~cm} \mathrm{H}_{2} \mathrm{O} ; \mathrm{p}<0,0001\right)$, índice BODE $(2,76 \pm 1,37$ vs $1,53 \pm 1,0 ; \mathrm{p}<0,0001)$, y escala de disnea mMRC $(2,54 \pm 0,75$ vs $1,18 \pm 0,56$; $\mathrm{p}<0,0001)$. Cuando se analizan por separado las variables síntomas, actividad e impacto del cuestionario respiratorio de Saint George, todas mejoraron significativamente. No se observaron cambios significativos en el $\mathrm{VEF}_{1}, \mathrm{IMC}_{\text {, y la flu- }}$ jometría al terminar el PRR. En cuanto al índice BODE, en la mayoría de los pacientes (84\%) se identificó una mejoría en al menos 1 punto en dicho índice (Figura 2), siendo los componentes de las variables disnea y TM6 los que influyeron principalmente en este cambio. Cuando se analizan los beneficios de la rehabilitación respiratoria en pacientes con EPOC leve-moderado $(n=21)$ comparado con EPOC grave-muy grave $(n=18)$, no se encontraron diferencias significativas en las variables de distancia recorrida del TM6, mMRC, IMC, BODE, PIMáx, ni flujometría, excepto en calidad de vida (Tabla 4), en donde la diferencia CSG final-CSG inicial ( $\triangle \mathrm{CSG}$ ), fue mayor en el grupo de pacientes con EPOC leve a moderado $(-32,7 \pm 9,6$ vs $-19,4 \pm 13,0, \mathrm{p}=0,001)$. Tam- 
Tabla 4. Análisis comparativo de paciente con EPOC leve-moderado vs grave-muy grave en relación al delta $(\Delta)$ de las variables capacidad física, disnea, fuerza muscular respiratoria, índice de masa corporal, índice BODE y calidad de vida $(n=39)$

\begin{tabular}{|lccc|}
\hline Variable & Pacientes GOLD I-II & Pacientes GOLD III-IV & p \\
$\Delta \mathrm{TM} 6, \mathrm{~m}$ & $57,7 \pm 40,2$ & $45,8 \pm 39,3$ & 0,36 \\
$\Delta$ mMRC, puntos & $-1,4 \pm 0,6$ & $-1,3 \pm 0,5$ & 0,36 \\
$\Delta$ Pimax, cmH $\mathrm{cm}_{2} \mathrm{O}$ & $10,1 \pm 7,6$ & $6,1 \pm 6,7$ & 0,09 \\
$\Delta \mathrm{IMC}, \mathrm{kg} / \mathrm{m}^{2}$ & $-0,3 \pm 0,8$ & $0 \pm 0$ & 0,13 \\
$\Delta$ Flujometría, L/min & $5,7 \pm 74,6$ & $32,2 \pm 30,2$ & 0,17 \\
$\Delta$ BODE, puntos & $-1,1 \pm 0,6$ & $-1,3 \pm 0,9$ & 0,46 \\
$\Delta$ CSG, puntos & $-32,7 \pm 9,6$ & $-19,4 \pm 13,0$ & 0,001 \\
\hline
\end{tabular}

Las cifras de la tabla representan el valor promedio de cada variable \pm su desviación estándar. Significado de las abreviaturas de las variables según Tabla 3.

Tabla 5. Análisis comparativo de paciente con EPOC GOLD I versus GOLD II en relación al delta ( $(\Delta)$ de las variables capacidad física, disnea, fuerza muscular respiratoria, índice de masa corporal, índice BODE y calidad de vida

\begin{tabular}{|lccc|}
\hline Variable & Pacientes GOLD I $(\mathbf{n}=\mathbf{8})$ & Pacientes GOLD II $(\mathbf{n}=\mathbf{1 4})$ & p \\
$\Delta$ TM6, m & $51,2 \pm 42,7$ & $58,9 \pm 42,7$ & NS \\
$\Delta$ mMRC, puntos & $-1,4 \pm 0,5$ & $-1,3 \pm 0,6$ & NS \\
$\Delta$ Pimax, cmH ${ }_{2} \mathrm{O}$ & $14,2 \pm 9,5$ & $7,4 \pm 4,8$ & NS \\
$\Delta$ IMC, $\mathrm{kg} / \mathrm{m}^{2}$ & $-0,3 \pm 0,8$ & $0 \pm 0$ & NS \\
$\Delta$ Flujometría, L/min & $32,5 \pm 30,1$ & $-6,4 \pm 87,3$ & NS \\
$\Delta$ BODE, puntos & $-1,13 \pm 0,4$ & $-1,07 \pm 0,7$ & NS \\
$\Delta$ CSG, puntos & $-36 \pm 9,8$ & $-28,9 \pm 11,1$ & NS \\
\hline
\end{tabular}

Las cifras de la tabla representan el valor promedio de cada variable \pm su desviación estándar. Significado de las abreviaturas de las variables según Tabla 3 .

poco, encontramos diferencias significativas en estas variables cuando se analizan y comparan los Grupos GOLD I vs GOLD II (Tabla 5).

\section{Discusión}

En el presente estudio se investigó el efecto de un programa de rehabilitación respiratoria realizado en la atención primaria de salud, en una zona rural de la Región Metropolitana en pacientes con EPOC, y se encontró una mejoría significativa en la disnea, calidad de vida, fuerza muscular, capacidad física, e índice BODE. Estos resultados son concordantes con lo publicado en la literatura médica que muestra beneficios similares con la RR, tanto si se realiza en el hospital, la atención primaria o en el domicilio del paciente $^{6-8,18-24}$. Por otra parte, hoy en día la rehabilitación respiratoria es un estándar de calidad en la atención de los pacientes con EPOC y las guías GOLD recomiendan que la rehabilitación respiratoria debería iniciarse muy precozmente en el tratamiento de esta enfermedad ${ }^{25,26}$. Esto, está fundamentado en que la reducción de la actividad física se presenta en etapas tempranas de la enfermedad ${ }^{27-29}$ y es un factor independiente de mayor frecuencia de hospitalizaciones y mortalidad ${ }^{30}$. Desafortunadamente, menos del $2 \%$ de los pacientes elegibles tienen acceso a rehabilitación respiratoria. En España ${ }^{31}$ por ejemplo, se estima que sólo el $0,3-0,6 \%$ de los pacientes con EPOC grave tiene acceso a RR, en Canadá ${ }^{32}$ el $1,2 \%$, y en Reino Unido $^{33}$ el $1,5 \%$. En Chile, no se cuenta con esta información a pesar que existen 530 salas ERA a lo largo del país, que están dotadas con kinesiólogos y además, se dispone de las guías EPOC-AUGE del Ministerio de Salud que incluyen como elementos terapéuticos no farmacológicos a la $\mathrm{RR}^{11}$, que sin embargo, aún 
no están implementadas. En efecto, una encuesta del Servicio de Salud Metropolitano Oriente, puso en evidencia que las actividades de rehabilitación respiratoria se realizaban en menos de la mitad de los centros de salud familiar, y sólo en el $25 \%$ de los centros los programas estaban bien estructurados ${ }^{34}$.

En nuestro estudio el índice BODE, un predictor de riesgo de muerte en pacientes con $\mathrm{EPOC}^{15}$, mejoró sustancialmente después de la RR. En la mayoría de los pacientes, 33/39 (84\%), se identificó una mejoría en al menos 1 punto en este índice. Esto es similar a lo encontrado en el estudio de Cote y Celli ${ }^{35}$, que compararon a pacientes EPOC con RR y otro grupo sin RR. Los autores concluyeron que la rehabilitación pulmonar mejora el índice BODE principalmente en los componentes de disnea y TM6, lo que se asocia con un mejor pronóstico. Se ha reportado, que este índice también es útil en la evaluación pronóstica de los pacientes con EPOC en la atención primaria ${ }^{36}$. Recientemente, el índice BODE modificado $^{37}$ al cual se agrega el consumo de oxígeno como quinta variable, fue comparado con el índice BODE tradicional, no obstante no mejoró la predicción pronóstica de mortalidad en pacientes con EPOC.

Al comparar los pacientes con EPOC levemoderado con grave-muy grave no observamos diferencias significativas en la mejoría de las variables de disnea, fuerza muscular respiratoria, capacidad de ejercicio, pero sí en calidad de vida donde el subgrupo de pacientes con EPOC leve a moderado tuvo un mejor resultado. Esto está de acuerdo con diversas publicaciones en que se demuestran que los beneficios de la RR es independiente de la gravedad de la enfermedad y puede ser efectiva, tanto en etapas tempranas como en etapas tardías de la EPOC $^{9,10,38}$.

En este estudio, los beneficios logrados con el PRR generaron tal impacto y motivación en los pacientes con EPOC, que voluntariamente formaron una Corporación de Rehabilitación Comunitaria donde continúan realizando actividad física supervisada por un kinesiólogo.

Nuestro estudio tiene algunas limitaciones. Primero, es preocupante el alto porcentaje de pacientes que abandonaron el PRR. Es conocido que muchos pacientes abandonan estos programas por reagudizaciones o problemas logísticos (transporte, dificultades económicas o caso social), estimándose una cifra superior al $20 \%{ }^{1}$. En nuestro estudio hubo un $28 \%$ de los pacientes que abandonaron el programa, siendo los problemas logísticos la principal causa. En segundo lugar, otra limitación es la falta de otros profesionales (psicólogo, nutricionista, asistente social, etc.) interesados en formar parte del equipo de rehabilitación, lo que permitiría evaluar otros aspectos del paciente con EPOC. Finalmente, la capacidad del PRR estuvo limitada por la disponibilidad de los recursos humanos, por lo cual cada grupo no fue mayor a 8-10 pacientes, cada 12 semanas.

\section{Conclusiones}

Los resultados de nuestro estudio son concordantes con otros estudios y demuestran los beneficios significativos obtenidos con la Rehabilitación Respiratoria en pacientes con EPOC, desde etapas iniciales a las avanzadas de la enfermedad. También, se demuestra que en Chile se puede implementar un PRR en la atención primaria utilizando implementos simples y a un bajo costo.

\section{Bibliografía}

1.- NICI L, DONNER C, WOUTERS E, ZUWALLACK R, AMBROSINO N, BOURBEAU J, et al. American Thoracic Society/European Respiratory Society statement on pulmonary rehabilitation. Am J Respir Crit Care Med 2006; 173: 1390-413.

2.- MURRAY C J L, LÓPEZ A D. Mortality by cause for eight regions of the world: Global Burden of Disease Study. Lancet 1997; 349: 1269-76.

3.- World Health Organization. Chronic Respiratory Diseases. Disponible en: http://www.who.int/respiratory/ copd/en/ (Consultado el 15 de mayo de 2011).

4.- SEPAR-ALAT. Guía de práctica clínica de diagnóstico y tratamiento de la enfermedad pulmonar obstructiva crónica. 2009. www.separ.es.

5.- MENEZES A M, PÉREZ-PADILLA R, JARDIM J R, MUIÑO A, LÓPEZ M V, VALDIVIA G, et al. PLATINO Team. Chronic obstructive pulmonary disease in five Latin American cities (the PLATINO study): a prevalence study. Lancet 2005; 366: 1875-81.

6.- RIES A L, BAULDOFF G S, CARLIN B W, CASABURI R, EMERY C, BMAHLER D, et al. Pulmonary rehabilitation: Joint ACCP/AACVPR evidence. Based Clinical Practice Guidelines. Chest 2007; 131: 4-42.

7.- BRITISH THORACIC SOCIETY. Standards of Care Subcommittee on Pulmonary Rehabilitation. Pulmonary rehabilitation. Thorax 2001; 56: 827-34.

8.- LACASSE Y, GOLDSTEIN R, LASSERSON T J, MARTIN S. Pulmonary rehabilitation for chronic obstructive pulmonary disease. Cochrane Database Syst Rev 2006: CD003793.

9.- BERRY M J, REJESKI W J, ADAIR N E, ZACCARO D. Exercise rehabilitation and chronic obstructive pul- 
monary disease stage. Am J Respir Crit Care Med 1999; 160: 1248-53.

10.- TAKIGAWA N, TADA A, SODA R, TAKAHASHI S, KAWATA N, SHIBAYAMA T, et al. Comprehensive pulmonary rehabilitation according to severity of COPD. Respir Med 2007; 101: 326-32.

11.- Ministerio de Salud de Chile. Guía Clínica de Enfermedad Pulmonar Obstructiva Crónica de Tratamiento Ambulatorio. Disponible en: http://www.redsalud.gov. cl/archivos/guiasges/enfermedadpulmonar.pdf (Consultado el 15 de mayo de 2011).

12.- JONES P W, QUIRK F H, BAVEYSTOCK C M, LITTLEJOHNS P. A self-complete measure of health status for chronic airflow limitation. The st. George's respiratory questionnaire. Am Rev Respir Dis 1992; 145: 1321-7.

13.- American Thoracic Society/European Respiratory Society. ATS/ERS statement on respiratory muscle testing. Am J Respir Crit Care Med 2002; 166: 518-624.

14.- American Thoracic Society. ATS statement: Guidelines for the six-minute walk test. Am J Respir Crit Care Med 2002; 166: 111-7.

15.- CELLI B R, COTE C G, MARÍN J M, CASANOVA C, MONTES DE OCA M, MÉNDEZ R A, et al. The bodymass index, airflow obstruction, dyspnea, and exercise capacity index in chronic obstructive pulmonary disease. N Engl J Med 2004; 350: 1005-12.

16.- BESTALL J C, PAUL E A, GARROD R, GARNHAM R, JONES P W, WEDZICHA J A. Usefulness of the Medical Research Council (MRC) dyspnea scale as a measure of disability in patients with chronic obstructive pulmonary disease. Thorax 1999; 54: 581-6.

17.- Global initiative for chronic obstructive lung disease. Global strategy for the diagnosis, management, and prevention of chronic obstructive pulmonary disease. Updated 2011. Disponible en: http://www.goldcopd.org/ guidelines-global-strategy-fordiagnosis-management. html (Consultado en mayo de 2012).

18.- WIJKSTRA P J, TEN VERGERT E M, VAN ALTENA R, OTTEN V, KRAAN J, POSTMA D S, et al. Long term benefits of rehabilitation at home on quality of life and exercise tolerance in patients with chronic obstructive pulmonary disease. Thorax 1995; 50: 824-8.

19.- STRIJBOS J H, POSTMA D S, VAN ALTENA R, GIMENO F, KOËTER G H. A comparison between an outpatient hospital-based pulmonary rehabilitation program and a home-care pulmonary rehabilitation program in patients with COPD. A follow-up of 18 months. Chest 1996; 109: 366-72.

20.- HERNÁNDEZ M T, RUBIO T M, RUIZ F O, RIERA H S, GIL R S, GÓMEZ J C. Results of a home-based training program for patients with COPD. Chest. 2000; 118: 106-14.

21.- FERRARI M, VANGELISTA A, VEDOVI E, FALSO M, SEGATTINI C, BROTTO E, et al. Minimally supervised home rehabilitation improves exercise capacity and health status in patients with COPD. Am J Phys Med Rehabil 2004; 83: 337-43.

22.- ELLIOT M, WATSON C, WILKINSON E, MUSK A W, LAKE F R. Short and long-term hospital and community exercise programmes for patients with chronic obstructive pulmonary disease. Respirology 2004; 9: 345-51.

23.- REGIANE RESQUETI V, GOROSTIZA A, GÁLDIZ J B, LÓPEZ DE SANTA MARÍA E, CASAN CLARÁ P, GÜELL ROUS R. Beneficios de un programa de rehabilitación respiratoria domiciliaria en pacientes con EPOC grave. Arch Bronconeumol 2007; 43: 599-604.

24.- GÜELL R, DE LUCAS P, GÁLDIZ J B, MONTEMAYOR T, RODRÍGUEZ GONZÁLEZ-MORO J M, GOROSTIZA A, et al. Home $v s$ hospital-based rehabilitation in COPD patients. A multicenter randomised trial. Arch Bronconeumol 2008; 44: 512-8.

25.- GÜELL M R, CEJUDO P, RODRÍGUEZ-TRIGO G, GÁLDIZ J B, CASOLIVE V, REGUEIRO M, et al. Standards for Quality Care in Respiratory Rehabilitation in Patients with Chronic Pulmonary Disease. Arch Bronconeumol 2012; 48: 396-404.

26.- RABE K F, HURD S, ANZUETO A, BARNES P J, BUIST S A, CALVERLEY P, et al. Global iniciative for chronic obstructive lung disease. Am J Respir Crit Care Med 2007; 176: 532-55.

27.- BABB T G, VIGGIANO R, HURLEY B, STAATS B, RODARTE J R. Effect of mild-to-moderate airflow limitation on exercise capacity. J Appl Physiol 1991; 70: 223-30.

28.- PITTA F, TROOSTERS T, SPRUIT M A, PROBST V S, DECRAMER M, GOSSELINK R. Characteristics of physical activities in daily life in chronic obstructive pulmonary disease. Am J Respir Crit Care Med 2005; 171: 972-7.

29.- PARADA A, KLAASSEN J, LISBOA C, SALDÍAS F, MENDOZA L, DÍAZ O. Reducción de la actividad física en pacientes con enfermedad pulmonar obstructiva crónica. Rev Med Chile 139: 1562-72.

30.- GARCÍA-AYMERICH J, LANGE P, BENET M, SCHNOHR P, ANTO J M. Regular physical activity reduces hospital admission and mortality in chronic obstructive pulmonary disease: A population based cohort study. Thorax 2006; 61: 772-8.

31.- MIRANDA G, GÓMEZ A, PLEGUEZUELOS E, CAPELLAS L. Rehabilitación respiratoria en España. Encuesta SORECAR. Rehabilitación (Madr) 2011; 45: 247-55.

32.- BROOKS D, SOTTANA R, BELL B, HANNA M, LAFRAMBOISE L, ELVANAYAGARAJAH S, et al. Characterization of pulmonary rehabilitation programs in Canada in 2005. Can Respir J 2007; 14: 87-92.

33.- YOHANNES A M, CONNOLY M J. Pulmonary rehabilitation programmes in the UK: a national representative survey. Clin Rehabil 2004; 18: 444-9.

34.- ARANCIBIA F, BELLO S, HINRICHSEN J. Manejo de 
las enfermedades respiratorias del adulto en los Centros de Salud Familiar del Servicio de Salud Metropolitano Oriente. Rev Chil Enferm Respir 2010; 4: A231-2.

35.- COTE C G, CELLI B R. Pulmonary rehabilitation and the BODE index in COPD. Eur Respir J 2005; 26: 6306.

36.- PUHAN M A, GARCÍA-AYMERICH J, FREY M, TER RIET G, ANTÓ J M, AGUSTÍ A G, et al. Expansion of the prognostic assessment of patients with chronic obstructive pulmonary disease: the updated BODE index and the ADO index. Lancet 2009; 374 (9691): 704-11.

37.- COTE C G, PINTO-PLATA V M, MARÍN J M, NEKACH H, DORDELLY L J, CELLI B R. The modified BODE index: validation with mortality in COPD. Eur Respir J. 2008; 32: 1269-74.

38.- ERGÜN P, KAYMAZ D, GÜNAY E, ERDOĞAN Y, TURAY U Y, DEMIR N, et al. Comprehensive outpatient pulmonary rehabilitation: treatment outcomes in early and late stages of chronic obstructive pulmonary disease. Ann Thorac Med 2011; 6: 70-6.

Correspondencia a:

Dr. Francisco Arancibia H.

Servicio de Medicina Respiratoria del Instituto Nacional del Tórax

José M. Infante 717, Providencia, Santiago.

Email: fearancibia@gmail.com 